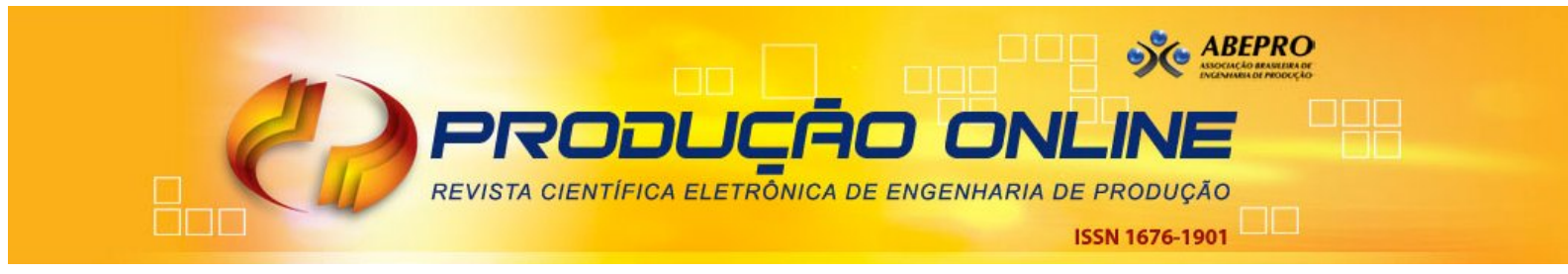

\title{
MODELOS DE PREVISÃO APLICADOS AO CONTROLE DE QUALIDADE COM DADOS AUTOCORRELACIONADOS ${ }^{1}$
}

\section{FORECAST MODEL APPLIED TO QUALITY CONTROL WITH AUTOCORRELATIONAL DATA}

\author{
Adriano Mendonça Souza* Email: adrianosm@gmail.com \\ Regiane Klidzio*Email: regianek@gmail.com \\ *Universidade Federal de Santa Maria, Santa Maria, RS
}

\begin{abstract}
Resumo: Essa pesquisa aborda os modelos de previsão, aplicados a processos produtivos industriais, com o objetivo de verificar a estabilidade do processo por meio de gráficos de controle, aplicado aos resíduos oriundos de modelagem linear. Os dados utilizados para análise referem-se ao teor de umidade, permeabilidade e resistência de compressão à verde (RCV), pertencentes ao processo de fundição de moldagem em areia verde da Empresa A, que atua no setor de fundição e usinagem, para os quais se ajustou um modelo de regressão dinâmica multivariada. Como as observações eram autocorrelacionadas, foi necessário buscar um modelo matemático com resíduos independentes e normalmente distribuídos. Os modelos matemáticos encontrados possibilitaram compreender o comportamento das variáveis, auxiliando na realização das previsões e na monitoração do referido processo. Assim, pode-se afirmar que o teor de umidade é muito instável em relação às outras variáveis.
\end{abstract}

Palavras-Chave: Séries temporais. Modelos lineares. Autocorrelação. Resíduos. Gráficos de controle.

Abstract: This research approaches the prediction models applied to industrial processes, in order to check the stability of the process by means of control charts, applied to residues from linear modeling. The data used for analysis refers to the moisture content, permeability and compression resistance to the green (RCV), belonging to the casting process of green sand molding in A Company, which operates in the casting and machining, for which dynamic multivariate regression model was set. As the observations were auto-correlated, it was necessary to seek a mathematical model that produces independent and identically distribuibed residues. The models found make possible to understand the variables behavior, assisting in the achievement of the forecasts and in the monitoring of the referred process. Thus, it can be stated that the moisture content is very unstable comparing to the others variables.

Keywords: Time Series. Linear Models. Autocorrelation. Residues. Control charts.

\section{INTRODUÇÃO}

Para que as empresas possam se consolidar no atual cenário competitivo, é preciso aumentar a qualidade de seus produtos e serviços. De acordo com Silveira e Werner (2011), a implementação do Controle Estatístico do Processo (CEP) tem

\footnotetext{
${ }^{1}$ Dissertação de Mestrado em Engenharia de Produção
}

Revista Produção Online, Florianópolis, SC, v.13, n. 4, p. 1276-1296, out./dez. 2013. 
sido utilizada para atingir esta meta, porém para obter eficiência é necessário priorizar os processos a serem monitorados.

Nesses termos, é preciso que o operador saiba identificar com clareza as variabilidades que estejam ocorrendo no processo, buscando com isso suas causas e tomando as devidas ações corretivas. Como consequência, à medida que a variabilidade é reduzida, ocorre também a melhoria da qualidade do produto (SILVA et al., 2007).

Segundo Montgomery (2004), as suposições geralmente necessárias para a utilização dos gráficos de controle são que os dados gerados pelo processo, quando sob controle, sejam distribuídos de acordo com a distribuição Normal com média $\mu$ e desvio padrão $\sigma$, e sejam independentes (RUSSO et al., 2008).

Frequentemente, as observações dos processos produtivos são autocorrelacionadas, o que afeta o desempenho dos gráficos de controle, resultando em muitos pontos fora dos limites (MONTGOMERY, 1997; TSAI et al., 2004). Nestes casos, há necessidade de se buscar novas soluções. Uma das abordagens utilizadas para tratar dados autocorrelacionados consiste em modelar diretamente a estrutura de correlação utilizando um modelo matemático de série temporal apropriado para remover a autocorrelação serial dos dados e então aplicar gráficos de controle aos resíduos desse modelo (ALWAN e ROBERTS, 1988; HARRIS e ROSS, 1991). Muitos autores têm discutido o assunto de como avaliar e tratar os efeitos da autocorrelação serial tais como: Mingoti e Yassukawa (2008), Claro et al. (2007), Moreira Junior e Ten Caten (2004) e Ramos e Ho (2003).

Em um processo produtivo quando há pelo menos três variáveis que devem ser analisadas conjuntamente, a análise univariada nem sempre é apropriada, pois o efeito de correlação entre as variáveis muitas vezes não é captado. E ainda, se existir correlação entre os erros das equações analisadas individualmente, é mais um motivo para se realizar uma estimação conjunta.

O objetivo dessa pesquisa é modelar um processo produtivo industrial autocorrelacionado com o fim de analisar a estabilidade do processo, por meio de gráficos de controle aplicado aos resíduos. Para atingir esse objetivo, será necessário realizar a modelagem linear com a finalidade de tratar a autocorrelação serial e, na sequência, comparar os efeitos da correlação utilizando gráficos de 
controle nos dados originais e nos resíduos sem a presença de autocorrelação serial.

\section{MODELOS DE VETORES AUTORREGRESSIVOS (VAR)}

Modelos de regressão dinâmica tornam-se eficiente, pois são capazes de detectar diversos instantes de tempo, isto é, valores presentes e defasados que afetam a variável de interesse. A metodologia consiste em estimar de forma individualizada cada equação, se o conjunto de variáveis se mostrar independente e se os resíduos oriundos destas equações também o forem. Caso exista um conjunto de variáveis a serem analisadas concomitantemente e se os resíduos de cada equação não forem independentes, pode-se então utilizar a metodologia do vetor autorregressivo (VAR), mantendo-se assim a característica dinâmica para representar o processo e garantindo a não correlação entre os resíduos.

A metodologia do vetor autorregressivo (VAR) possibilita uma estimação conjunta dos parâmetros, em que as inter-relações são consideradas, e o comportamento dinâmico das variáveis é capturado, fornecendo uma estrutura entre as variáveis de entrada e de saída do sistema (SOUZA et al., 2004a). Dessa maneira, torna-se possível aumentar a acurácia das estimativas do modelo, utilizando-se informações adicionais fornecidas pelas inter-relações.

No VAR, cada variável do sistema de equações lineares dinâmicas é escrita como uma combinação linear de suas defasagens, juntamente com as defasagens das variáveis endógenas de outras equações (BAYER et al., 2007) e em função de um erro não-correlacionado, e todas as variáveis que pertencem ao sistema possuem o mesmo número de defasagens, representadas por $p$, estas determinam a ordem do modelo, que, genericamente, é representado por $\operatorname{VAR}(p)$, conforme a equação (1) (SOUZA et al., 2004b). Esses modelos são amplamente discutidos por Lütkepohl (1991), Maddala (1992), Charemza e Deadman (1997), Reinsel (1993) e Hamilton (1994).

$$
Z_{t}=v+\varphi_{1} Z_{t-1}+\ldots+\varphi_{p} Z_{t-p}+\varepsilon_{t}
$$

onde $Z_{t}$ é um vetor aleatório, $\varphi_{i}$ é a matriz dos coeficientes, $v$ é o vetor dos interceptos, o qual permite que a média do processo seja diferente de zero e $\varepsilon_{t}$ é o vetor ruído branco. Este processo ruído branco garante que os erros sejam 
independentes e identicamente distribuídos (iid), indicando a ausência de qualquer correlação serial entre os erros, ou seja, que os resíduos serão homoscedásticos.

Segundo Gatu e Kontoghiorghes (2006), a metodologia de vetor autorregressivo (VAR) pode ser aplicada como um modelo de regressões aparentemente não correlacionadas, que vem do termo inglês Seegmingly Unrelated Regression (SUR). Esse método possibilita que cada equação tenha sua própria forma funcional, levando em consideração apenas a correlação existente entre os resíduos dessas equações (HAMILTON, 1994; HILL et al., 1999; MADDALA, 1992; SOUZA et al., 2004a). E, leva em consideração a apenas a correlação existente entre os resíduos das equações (SALLES, 2006).

De acordo com Zellner e Theil (1962), a aplicação do estimador SUR que também pode ser entendida como o Método dos Mínimos Quadrados Ordinários em Três Estágios (MQO3E) admite a estimação dos parâmetros das equações simultâneas, possibilitando diferentes restrições na estrutura das equações o que possibilita o ganho de eficiência nas estimativas. E, segundo Creel e Farell (1996) o estimador SUR pode ser substancialmente mais eficiente que o método de Mínimos Quadrados Ordinários (MQO), Brons et al. (2008) corroboram a ideia dizendo que o SUR gera resultados mais precisos.

O procedimento de estimação do MQO3E considera todas as equações em conjunto, e não separadamente. Os três estágios deste método são:

a) estimar as equações separadamente utilizando mínimos quadrados;

b) utilizar os resíduos de mínimos quadrados, do passo anterior, para estimar as variâncias e a covariância dos erros;

c) utilizar as estimativas das variâncias dos erros para estimar as equações conjuntamente.

Segundo Hill et al. (1999), o emprego do MQO3E usa a matriz dos momentos dos erros para estimar todos os coeficientes envolvendo todo o sistema simultaneamente. Sendo assim, o método possui todas as informações, de modo que, se a estrutura de covariância dos erros não for zero, a estimação dos coeficientes de qualquer equação ganha em eficiência tão logo outras equações sejam identificadas.

O sistema de equações multivariado após a estimação conjunta deve apresentar um processo ruído branco de forma que os resíduos sejam iid, 
garantindo a ausência de autocorrelação. Além disso, é necessário que os resíduos sejam homoscedásticos, seguindo a distribuição Normal, desta forma estando de acordo com as necessidades para a aplicação de gráficos de controle. Então, realizada a análise dos resíduos, e tendo todas estas hipóteses satisfeitas, o modelo e os resíduos podem ser utilizados em etapas posteriores, como previsões, por exemplo (HILL et al., 1999; GUJARATI, 2000).

\section{METODOLOGIA}

Nesta pesquisa ex-post-facto será realizado um estudo de controle de qualidade utilizando-se um modelo de regressão dinâmica multivariada, tendo em vista que as variáveis sob estudo são inter-relacionadas. Ao encontrar um modelo matemático para cada uma das variáveis envolvidas, construíram-se os respectivos gráficos de controle utilizando-se os resíduos do modelo estimado com o objetivo de verificar a estabilidade do processo, identificando as possíveis variáveis causadoras dessa instabilidade.

As variáveis utilizadas nesta pesquisa são o teor de umidade, permeabilidade e resistência de compressão à verde (RCV), abrangendo o período de 29 de abril de 2008 a 30 de junho de 2009, totalizando 319 observações, com coletas diárias considerando os dias que ocorre o processo de fundição de moldagem em areia verde.

Do total das 319 observações, 313 foram utilizadas para estimar o modelo dinâmico multivariado, as seis últimas observações foram utilizadas para validar o modelo estimado.

Como o processo produtivo apresenta características multivariadas, utiliza-se a modelagem VAR por meio do Método dos Mínimos Quadrados Ordinários em Três Estágios (MQO3E). Para a estimação dos valores previstos para cada equação, onde se espera que estes resíduos possam ser utilizados para avaliação do processo, as estatísticas utilizadas para a validação das previsões serão os critérios MAPE e $U$ de Theil.

A análise da estabilidade do processo será realizada utilizando os gráficos de controle para medidas individuais $(\bar{x})$ e amplitude móvel $(\overline{M R})$, aplicado aos 
resíduos oriundos da modelagem VAR, utilizando-se subgrupos amostrais de tamanho unitário.

Os softwares utilizados foram: Statistica 7.0, EViews 6.0 e planilha eletrônica. Ressalta-se que, para interpretação dos resultados dos testes estatísticos aplicados, adotou-se um nível de significância de 5\%.

\section{ANÁLISE DOS RESULTADOS}

Essa seção apresenta a análise dos resultados mediante a aplicação da metodologia proposta em conjuntos de dados reais referentes ao processo produtivo industrial da Empresa A, especializada na fundição de ferro fundido cinzento e nodular e usinagem, que por questões éticas o nome da empresa não será revelado. O processo de fundição utilizado na empresa é a moldagem em areia verde, também conhecido como areia sintética.

Basicamente, o processo de fundição pode ser resumido nas seguintes operações: fabricação do molde, elaboração do metal e vazamento do metal. Um molde é construído para cada peça a ser fundida e, subsequentemente, ele é rompido para que a peça fundida possa ser removida.

Analisando os defeitos que podem ocorrer na peça fundida juntamente com o engenheiro mecânico da empresa, verificou-se que muitos deles são causados pelas variações nas especificações das características da areia verde. Por este motivo, justifica-se fazer uma estimação multivariada das variáveis: teor de umidade (\%), permeabilidade (AFS) e resistência de compressão à verde (L/POL $\left.{ }^{2}\right)$, as quais são consideradas geradoras de instabilidades. Essas variáveis estão intimamente relacionadas, e, possuem especificações determinadas pela Engenharia de Produção e Engenharia Técnica.

\subsection{Análise descritiva}

Após a aquisição dos dados, é preciso conhecer o comportamento das variáveis no decorrer do período analisado. A Tabela 1 apresenta as estatísticas descritivas pertinentes ao estudo do teor de umidade, permeabilidade e RCV. Com a análise descritiva dessas variáveis, verificou-se, pelo coeficiente de variação (CV), 
que as médias das variáveis são representativas, pois o seu valor percentual é muito abaixo de 30\% (MUROLO et al., 1999).

Tabela 1 - Estatísticas descritivas para as variáveis Teor de umidade, Permeabilidade e RCV da Empresa A no período de 29/04/2008 a 30/06/2009.

\begin{tabular}{l|ccc}
\hline Estatísticas & Teor de umidade & Permeabilidade & RCV \\
\hline Média & 3,490 & 155,893 & 18,737 \\
Mediana & 3,500 & 160,000 & 19,000 \\
Desvio padrão & 0,242 & 11,239 & 1,757 \\
Coeficiente de variação & 6,930 & 7,210 & 9,378 \\
Assimetria & $-0,148$ & 0,009 & 0,960 \\
Curtose & $-0,222$ & $-0,159$ & 2,554 \\
Mínimo & 2,900 & 130,000 & 15,000 \\
Máximo & 4,200 & 190,000 & 27,000 \\
\hline
\end{tabular}

Embora a análise descritiva revele o comportamento do processo e a Tabela 1 seja autoexplicativa e o CV seja considerado baixo, é necessário investigar sua estabilidade por meio dos gráficos de controle apresentados nas Figuras 2, 3, 4, 5, 6 e 7, os quais avaliarão a estabilidade do processo. É importante lembrar que, em um processo produtivo quanto menor a variabilidade melhor será a qualidade dos produtos finais.

Inicialmente, estimaram-se as equações independentemente por meio do método de MQO, analisando as variáveis individualmente. Com os resíduos oriundos das equações, calculou-se a matriz de correlação, a qual revelou que existe correlação, indicando ligação entre as variáveis por meio de seus resíduos.

Como esta correlação foi detectada, é aconselhável fazer uma análise conjunta, de modo a fornecer informações relevantes sobre a relação existente entre as variáveis a partir do comportamento multivariado. Esse pressuposto comprova a estimação de um modelo matemático utilizando equações aparentemente não correlacionadas.

\subsection{Estimação do modelo de regressão multivariado}

As séries temporais correspondentes às variáveis sob estudo, apresentam-se inter-relacionados, portanto, não é possível a utilização direta destes valores observados pelos gráficos de controle, pois os pressupostos de normalidade, homocedasticidade e independência são violados. Utiliza-se então um modelo 
matemático que possibilita uma estimação conjunta, considerando as relações existentes entre as variáveis e as relações dinâmicas que ocorrem entre elas, obtendo-se uma equação para cada variável, que proporcionem resíduos iid para, em seguida, aplicar os gráficos de controle nos resíduos desse modelo. Encontra-se o modelo de regressão dinâmica multivariada que representasse as variáveis utilizando o método de estimação SUR.

Apresentam-se a seguir, as equações estimadas para o conjunto de variáveis, levando em consideração uma defasagem inicial de ordem três, onde as defasagens não significativas foram eliminadas. As equações foram ajustadas por meio do MMQ3E.

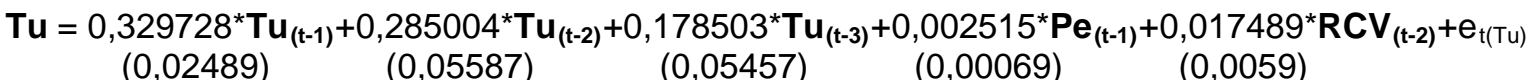

$$
\begin{aligned}
& P e=0,308247 * P_{(t-1)}+0,337874 * P_{(t-2)}+0,239161 * P e_{(t-3)}+0,945208^{*} R_{C V} V_{(t-3)}+e_{t(P e)} \\
& \begin{array}{llll}
(0,05347) \quad(0,05314) & (0,05271) & (0,276)
\end{array} \\
& \mathbf{R C V}=0,15892^{\star} \mathbf{T u}_{(\mathrm{t}-2)}+0,022881^{*} \mathbf{P e}_{(\mathrm{t}-3)}+0,198212^{*} \mathbf{R C V}_{(\mathrm{t}-1)}+0,20179 * \mathbf{R C V}_{(\mathrm{t}-2)}+0,210247^{*} \mathbf{R C V}_{(\mathrm{t}-}
\end{aligned}
$$

onde: Tu representa a variável teor de umidade; $\boldsymbol{P e}$ representa a variável permeabilidade e $\boldsymbol{R} C \boldsymbol{V}$ representa a variável resistência de compressão à verde.

As equações estimadas apresentaram características de ruído branco, o que possibilita a utilização dos resíduos para futuras análises. A Figura 1 exibe a Função de Autocorrelação (FAC) para o teor de umidade, a permeabilidade e o RCV, comprovando essa afirmação. 
Figura 1 - FAC das variáveis: teor de umidade, permeabilidade e RCV
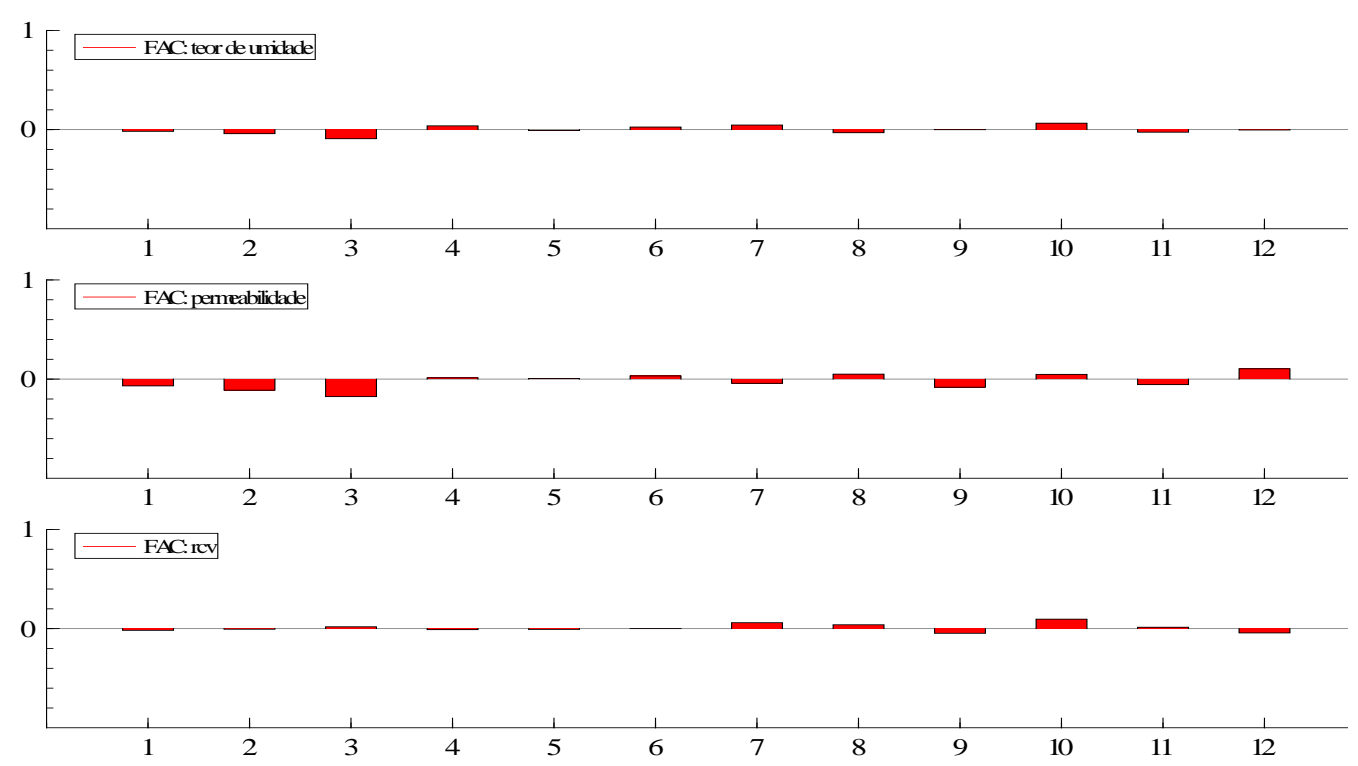

Além de o modelo matemático eliminar a autocorrelação das variáveis em estudo, é importante analisar a normalidade e a homocedasticidade dos resíduos com o intuito de avaliar se os pressupostos básicos dos gráficos de controle são atendidos.

Para comprovar que o modelo matemático de equação dinâmica multivariada é adequado, apresenta-se, na Tabela 2, um diagnóstico dos resíduos do modelo estimado.

As hipóteses testadas são as seguintes:

$\mathrm{H}_{0}$ : Homocedasticidade dos resíduos;

$\mathrm{H}_{1}$ : Heterocedasticidade dos resíduos.

Tabela 2 - Diagnóstico dos resíduos multivariados

\begin{tabular}{|c|c|c|c|}
\hline Testes & Resultado & & Interpretação \\
\hline Teste de normalidade & $\mathrm{Chi}^{2}(6)=$ & {$[0,404]^{*}$} & Aceita $\mathrm{H}_{0}$ \\
\hline $\begin{array}{l}\text { Teste de White sem os } \\
\text { produtos cruzados }\end{array}$ & $F(108,1623)=0,772$ & {$[0,959]^{\star}$} & Aceita $\mathrm{H}_{0}$ \\
\hline $\begin{array}{l}\text { Teste de White com os } \\
\text { produtos cruzados }\end{array}$ & $F(324,1477)=1,127$ & {$[0,079]^{\star}$} & Aceita $\mathrm{H}_{0}$ \\
\hline
\end{tabular}

Analisando o p-valor para cada teste, constatou-se que os resultados dos testes empregados estão na região de aceitação da hipótese nula $\left(\mathrm{H}_{0}\right)$, ou seja, os resíduos são homocedásticos. Sendo assim, o teste de normalidade apontou 
evidências que os resíduos do modelo matemático são normais, e, os testes de heterocedasticidade, Testes de White, assinalaram que os mesmos são homocedásticos.

Os testes apresentados na Tabela 3, que levam em conta as variáveis individualmente, confirmaram os resultados obtidos na Tabela 2.

Para comprovar a presença de efeitos $\mathrm{ARCH}$, testam-se as seguintes hipóteses:

$$
\begin{aligned}
& \mathrm{H}_{0}: \alpha_{i}=0 \text {, ou seja, não existem efeitos } \mathrm{ARCH} ; \\
& \mathrm{H}_{1}: \alpha_{i} \neq 0 \text {, ou seja, existem efeitos } \mathrm{ARCH} \text {. }
\end{aligned}
$$

Tabela 3 - Diagnóstico dos resíduos individuais

\begin{tabular}{l|l|l|c}
\hline Testes & \multicolumn{1}{|c|}{ Variáveis } & \multicolumn{1}{|c}{ Resultado } & Interpretação \\
\hline \multirow{2}{*}{ Teste ARCH-LM } & Teor de umidade & $F(1,304)=0,108[0,742]^{\star}$ & Aceita $\mathrm{H}_{0}$ \\
& Permeabilidade & $F(1,304)=0,173[0,678]^{\star}$ & Aceita $\mathrm{H}_{0}$ \\
& RCV & $F(1,304)=2,226[0,137]^{\star}$ & Aceita $\mathrm{H}_{0}$ \\
\hline Teste de White & Teor de umidade & $F(18,287)=0,480[0,965]^{*}$ & Aceita $\mathrm{H}_{0}$ \\
sem os produtos & Permeabilidade & $F(18,287)=1,279[0,201]^{*}$ & Aceita $\mathrm{H}_{0}$ \\
cruzados & RCV & $F(18,287)=0,511[0,952]^{*}$ & Aceita $\mathrm{H}_{0}$ \\
\hline Teste de White & Teor de umidade & $F(54,251)=0,666[0,963]^{\star}$ & Aceita $\mathrm{H}_{0}$ \\
com os produtos & Permeabilidade & $F(54,251)=1,433[0,052]^{*}$ & Aceita $\mathrm{H}_{0}$ \\
cruzados & RCV & $F(54,251)=1,059[0,376]^{\star}$ & Aceita $\mathrm{H}_{0}$ \\
\hline
\end{tabular}

Observando-se os p-valores dos testes apresentados na Tabela 3, constatouse que o Teste ARCH-LM e os Testes de White estão na região de aceitação $\left(\mathrm{H}_{0}\right)$, indicando que não existem efeitos $\mathrm{ARCH}$, ou seja, que os resíduos do modelo são homocedásticos. Sendo assim, o modelo de regressão dinâmica multivariada não apresenta heterocedasticidade condicional em nível de significância de 5\%, ou seja, há evidências que os resíduos apresentam variância constante, conhecida por homocedasticidade.

Mediante os resultados obtidos pelo diagnóstico dos resíduos constatou-se que os mesmos atendem os pressupostos básicos para aplicação dos gráficos de controle. Antes de verificar a estabilidade do processo através desses gráficos, apresenta-se, na Tabela 4, os valores originais e previstos. 
Tabela 4 - Período, valores originais e valores previstos para as variáveis Teor de umidade, Permeabilidade e RCV da Empresa A

\begin{tabular}{lcccccc}
\hline & \multicolumn{2}{c}{$\begin{array}{c}\text { Previsão para o } \\
\text { Teor de umidade }\end{array}$} & \multicolumn{2}{c}{$\begin{array}{c}\text { Previsão para a } \\
\text { Permeabilidade }\end{array}$} & \multicolumn{2}{c}{$\begin{array}{c}\text { Previsão para a } \\
\text { RCV }\end{array}$} \\
\hline Período & Originais & Previstos & Originais & Previstos & Originais & Previstos \\
\hline $24 / 6 / 2009$ & 3,500 & 3,286 & 140,000 & 156,070 & 19,000 & 18,012 \\
$25 / 6 / 2009$ & 3,400 & 3,331 & 155,000 & 155,265 & 19,000 & 18,574 \\
$26 / 6 / 2009$ & 3,600 & 3,347 & 165,000 & 154,425 & 18,000 & 18,251 \\
$27 / 6 / 2009$ & 3,500 & 3,353 & 145,000 & 154,412 & 20,000 & 18,271 \\
$28 / 6 / 2009$ & 3,400 & 3,361 & 150,000 & 154,463 & 20,000 & 18,331 \\
$30 / 6 / 2009$ & 3,400 & 3,369 & 155,000 & 153,968 & 17,000 & 18,267 \\
\hline
\end{tabular}

Conforme mencionado anteriormente, o modelo estimado foi elaborado com 313 observações. As últimas seis ficaram de fora da amostra com o objetivo de avaliar o modelo matemático. Sendo assim, a previsão ocorre da observação 314 à observação 319.

Os valores dos critérios de avaliação MAPE(\%) e U de Theil para as variáveis sob estudo são:

- Teor de umidade: $\operatorname{MAPE}(\%)=3,574$ e $U$ de Theil= 0,954;

- Permeabilidade: MAPE(\%)= 4,698 e U de Theil= 0,522;

- RCV: MAPE(\%)= 5,547 e U de Theil= 0,745.

Os critérios de avaliação comprovaram estatisticamente que o modelo estimado apresenta uma previsão melhor que a previsão ingênua ou trivial.

\subsection{Análise da estabilidade do processo}

Nesta etapa, o objetivo é monitorar o processo de fundição de moldagem em areia verde, a partir do modelo de regressão multivariada, para verificar sua estabilidade e tentar identificar as variáveis responsáveis pela instabilidade utilizando os gráficos de controle.

A análise da estabilidade do processo foi realizada utilizando os gráficos de controle para medidas individuais $(\bar{x})$ e amplitude móvel $(\overline{M R})$, pois as amostras das variáveis são de tamanho unitário. 
Apresentam-se a seguir, os gráficos de controle $\bar{x}$ e $\overline{M R}$ nas Figuras 2, 3, 4, 5,6 e 7 usando os dados originais para mostrar como está o comportamento das variáveis: teor de umidade, permeabilidade e RCV.

Figura 2 - Gráfico $\bar{x}$ do teor de umidade, considerando os dados originais

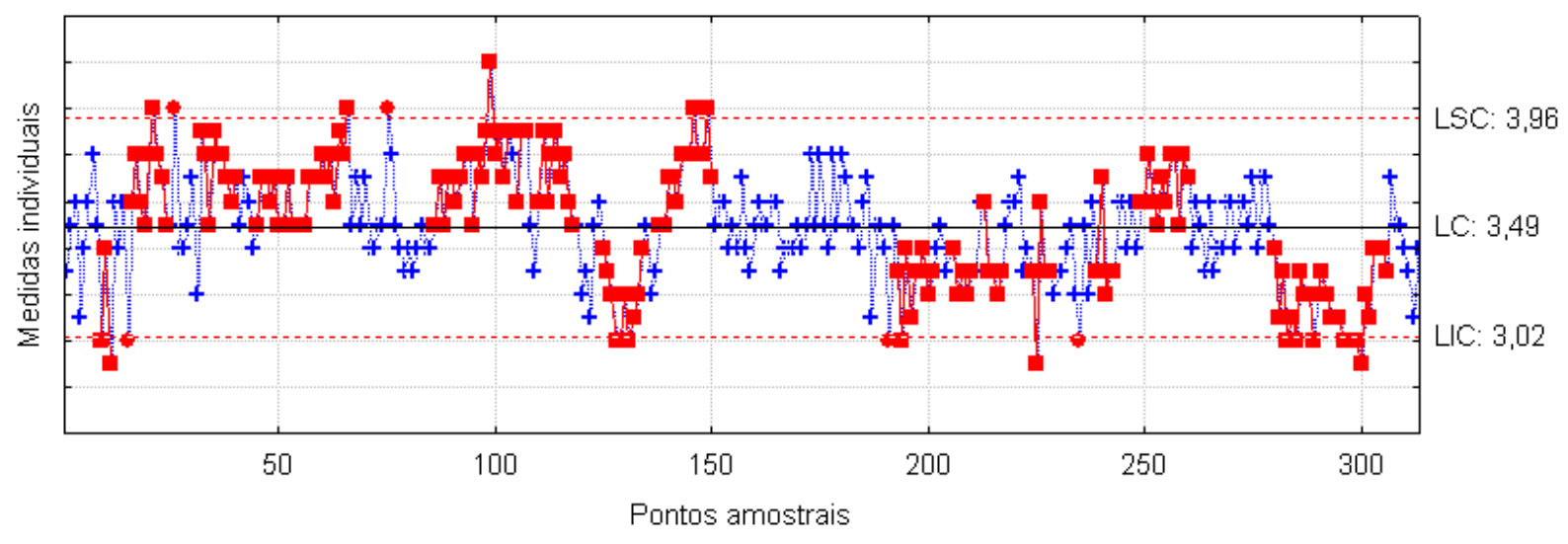

Figura 3 - Gráfico $\overline{M R}$ do teor de umidade, considerando os dados originais

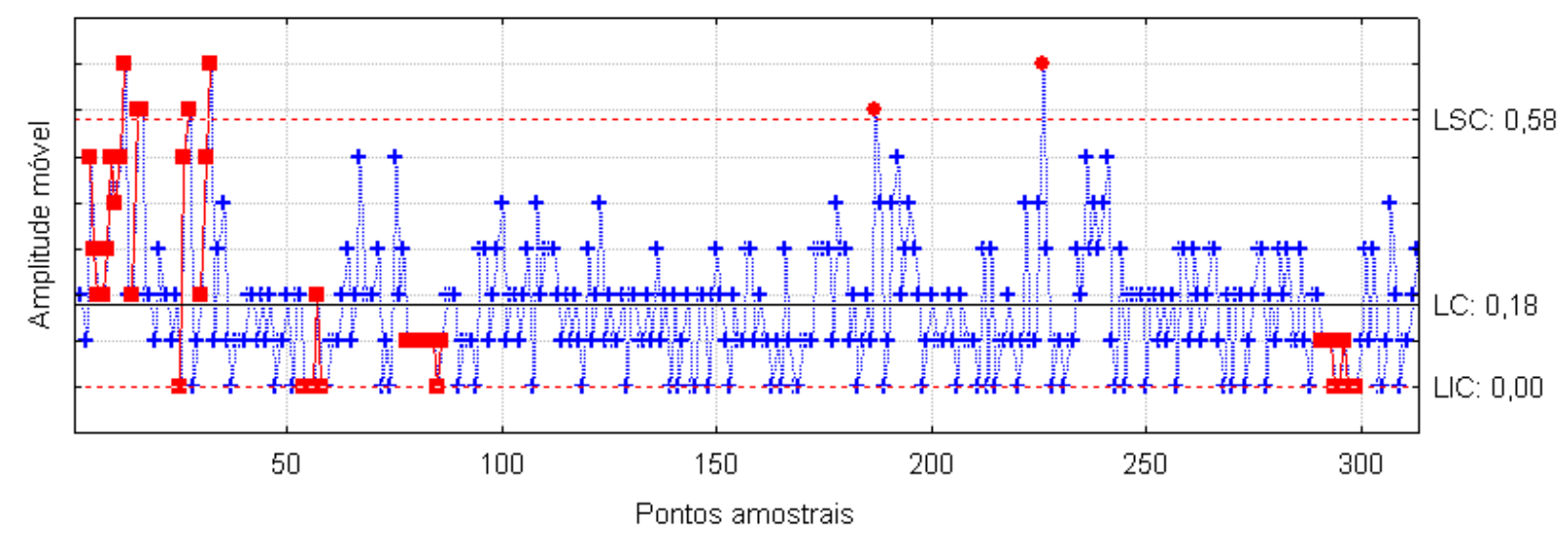

Figura 4 - Gráfico $\bar{X}$ da permeabilidade, considerando os dados originais

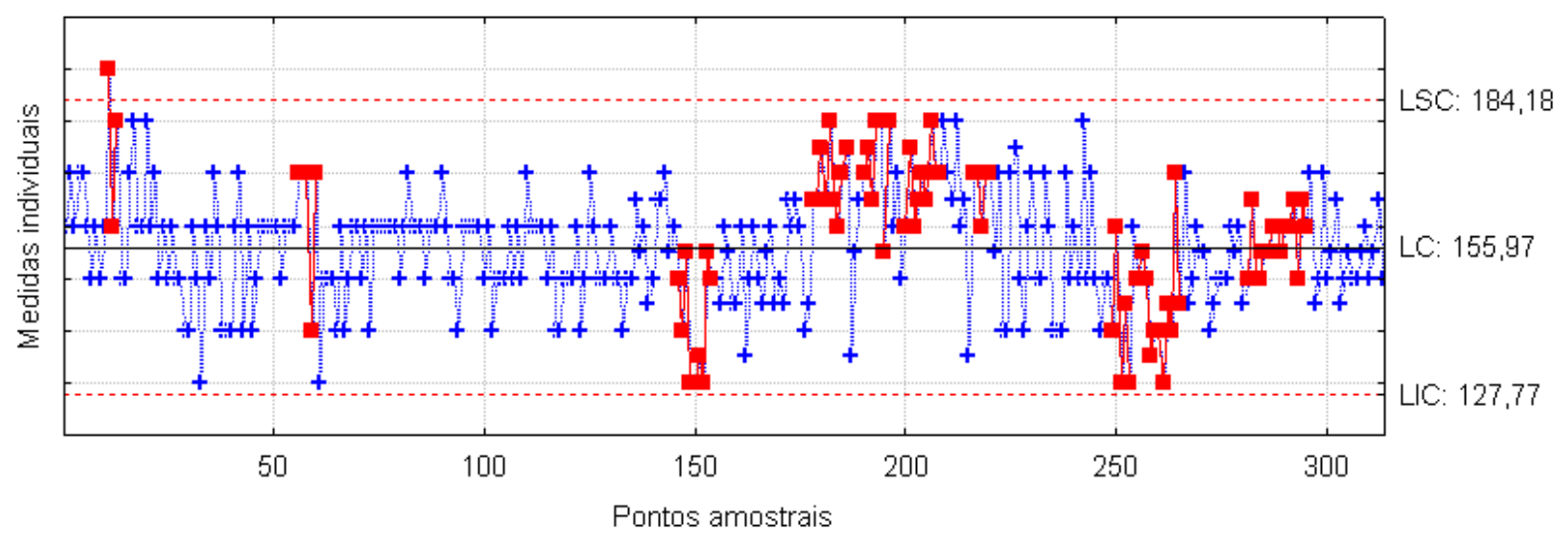

Revista Produção Online, Florianópolis, SC, v.13, n. 4, p. 1276-1296, out./dez. 2013. 
Figura 5 - Gráfico $\overline{M R}$ da permeabilidade, considerando os dados originais

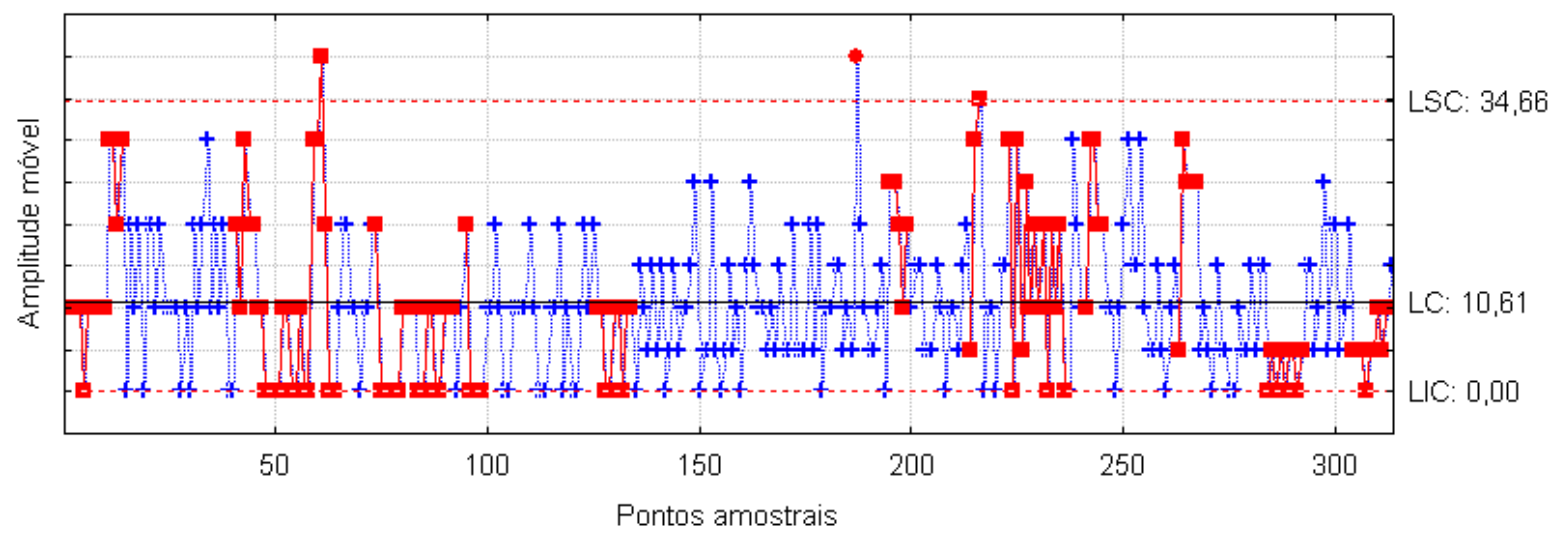

Figura 6 - Gráfico $\bar{x}$ da RCV, considerando os dados originais

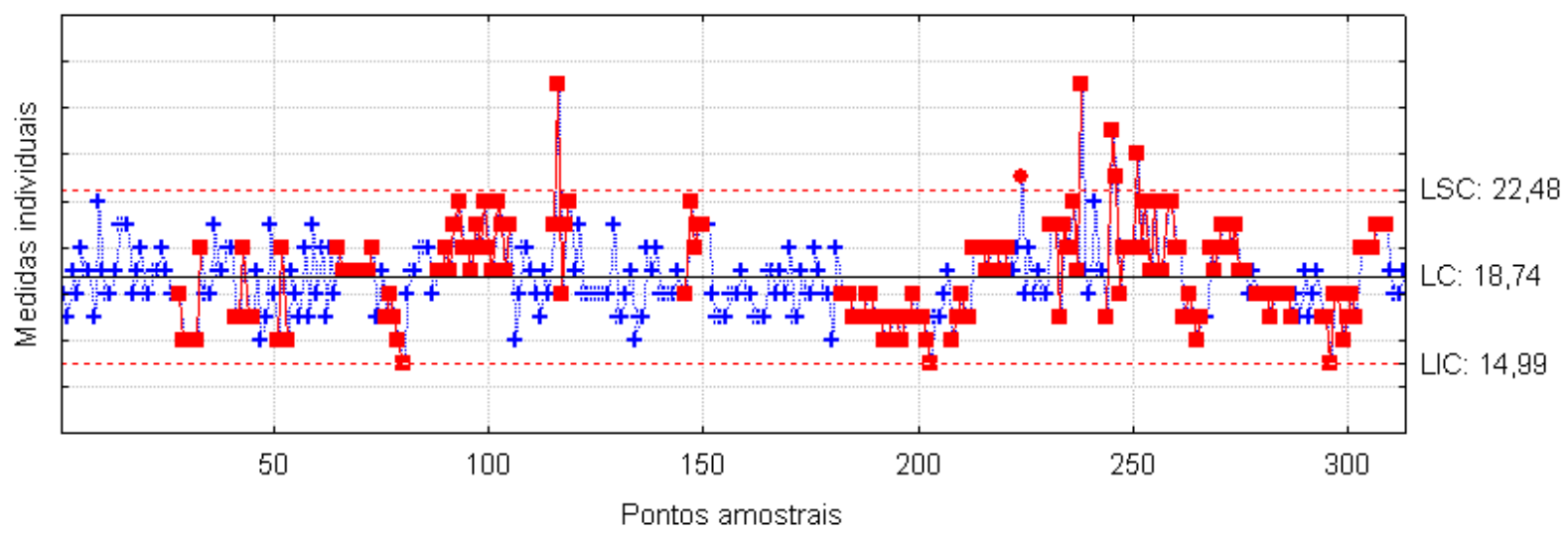

Figura 7 - Gráfico $\overline{M R}$ da RCV, considerando os dados originais

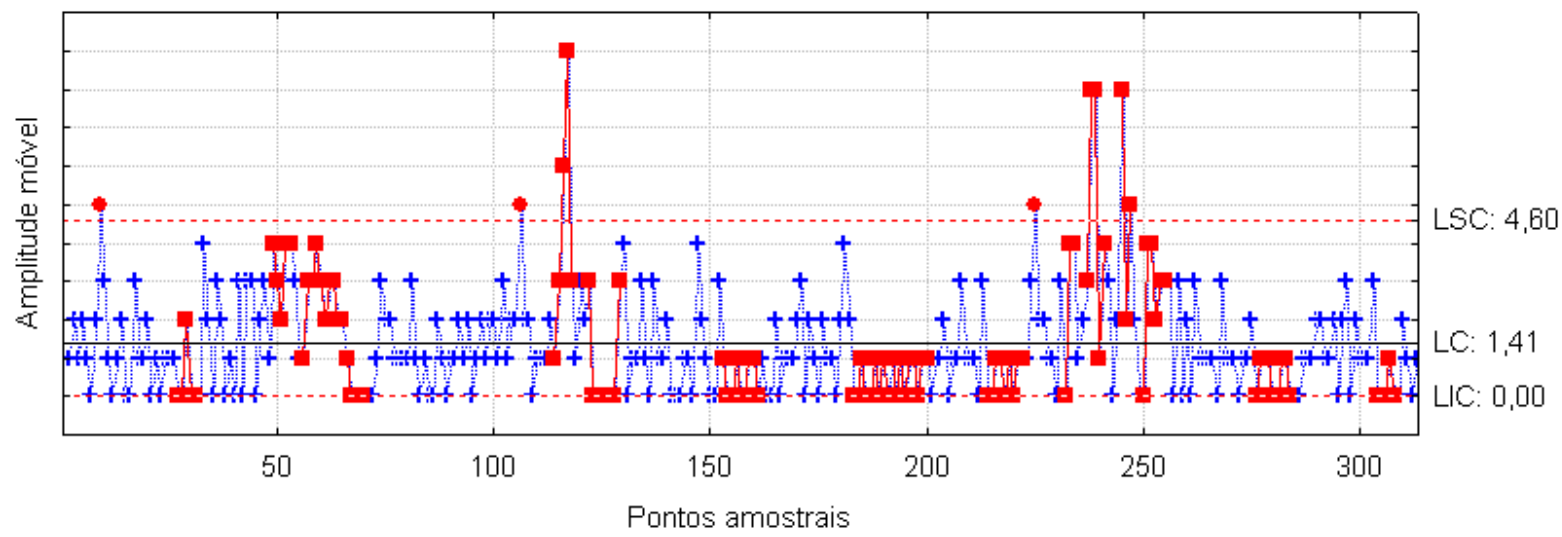


Observando-se a Figuras 2, 3, 4, 5 e 6 percebe-se a elevada quantidade pontos amostrais fora de controle estatístico. Ao realizar outra análise, simulando a exclusão dos pontos amostrais fora de controle, tem-se outra visão da instabilidade que está ocorrendo no processo.

A partir dos resultados obtidos, justifica-se que as técnicas convencionais do CEP não podem ser utilizadas diretamente nos dados originais, ou seja, é necessário um pré-tratamento na variável de interesse para remover a autocorrelação serial, pois a mesma pode inserir pontos amostrais fora de controle, quando na verdade o processo pode estar sob controle estatístico (SOUZA et al., 2012).

Para sanar o efeito de autocorrelação serial e a correlação entre as variáveis, estimou-se um modelo de regressão dinâmica multivariada, atendendo perfeitamente os pressupostos para a aplicação de gráficos de controle. Sendo assim, foram utilizados os resíduos do modelo estimado para traçar os gráficos $\bar{x}$ e $\overline{M R}$, tendo como auxílio na decisão a aplicação dos runs tests.

Pelas Figuras 8, 9, 10, 11, 12 e 13 é possível identificar a ocorrência de padrões especiais, caracterizando o comportamento do processo de fundição de moldagem em areia verde quanto a sua estabilidade e/ou instabilidade de modo que seja feito ou não um ajuste no processo.

Figura 8 - Gráfico $\bar{X}$ aplicado ao resíduo do teor de umidade

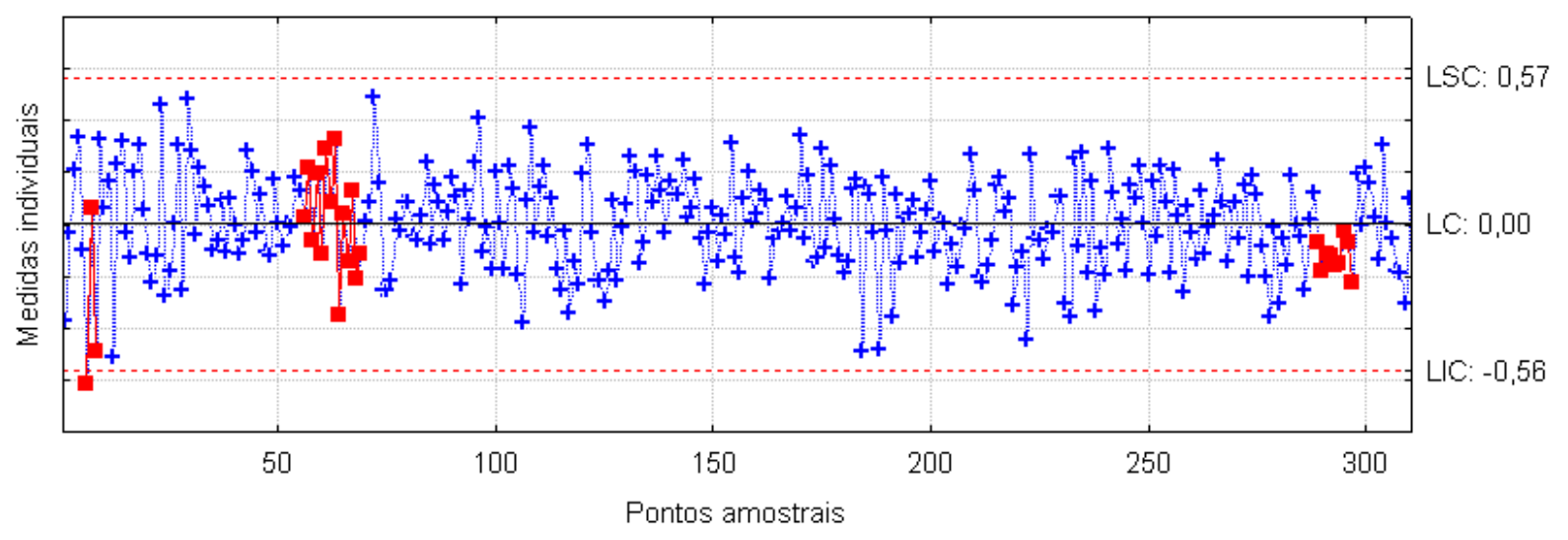


Figura 9 - Gráfico $\overline{M R}$ aplicado ao resíduo do teor de umidade

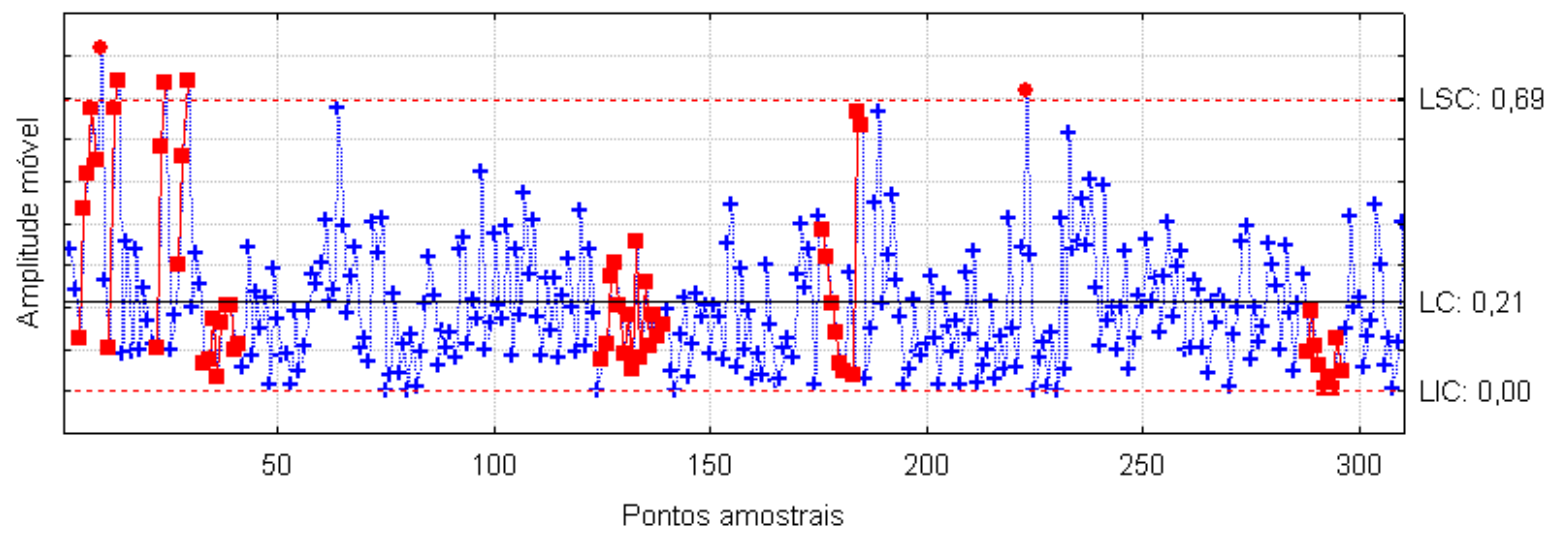

Figura 10 - Gráfico $\bar{X}$ aplicado ao resíduo da permeabilidade

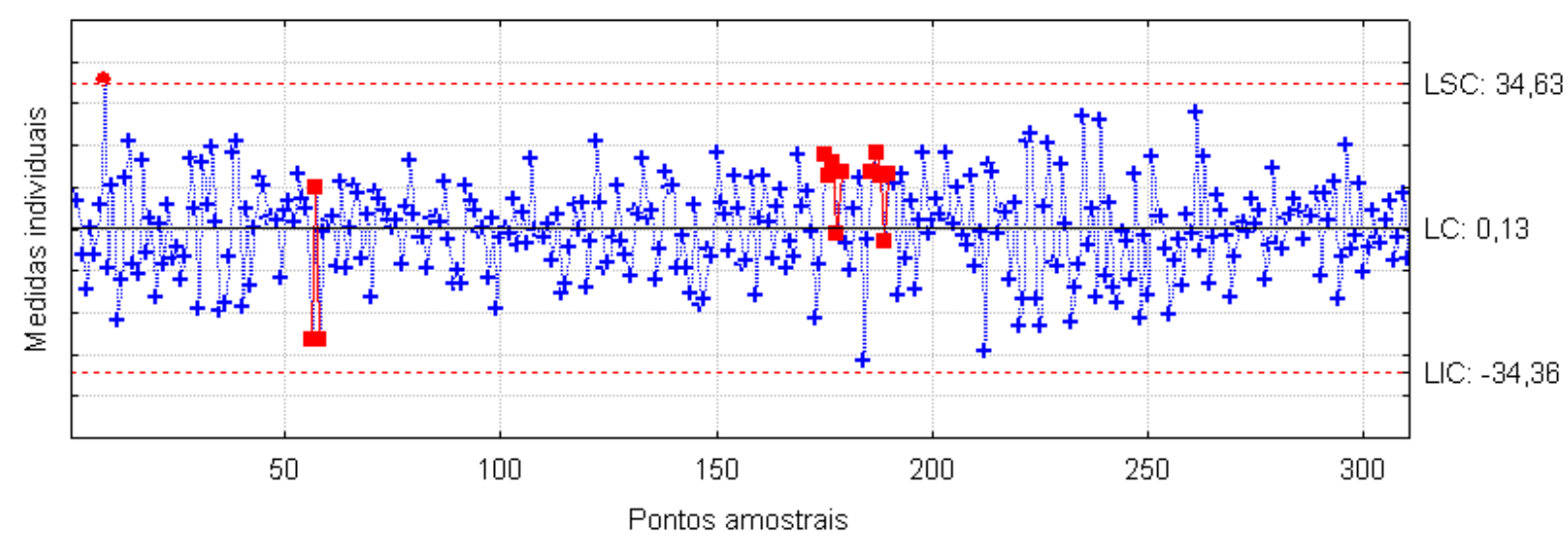

Figura 11 - Gráfico $\overline{M R}$ aplicado ao resíduo da permeabilidade

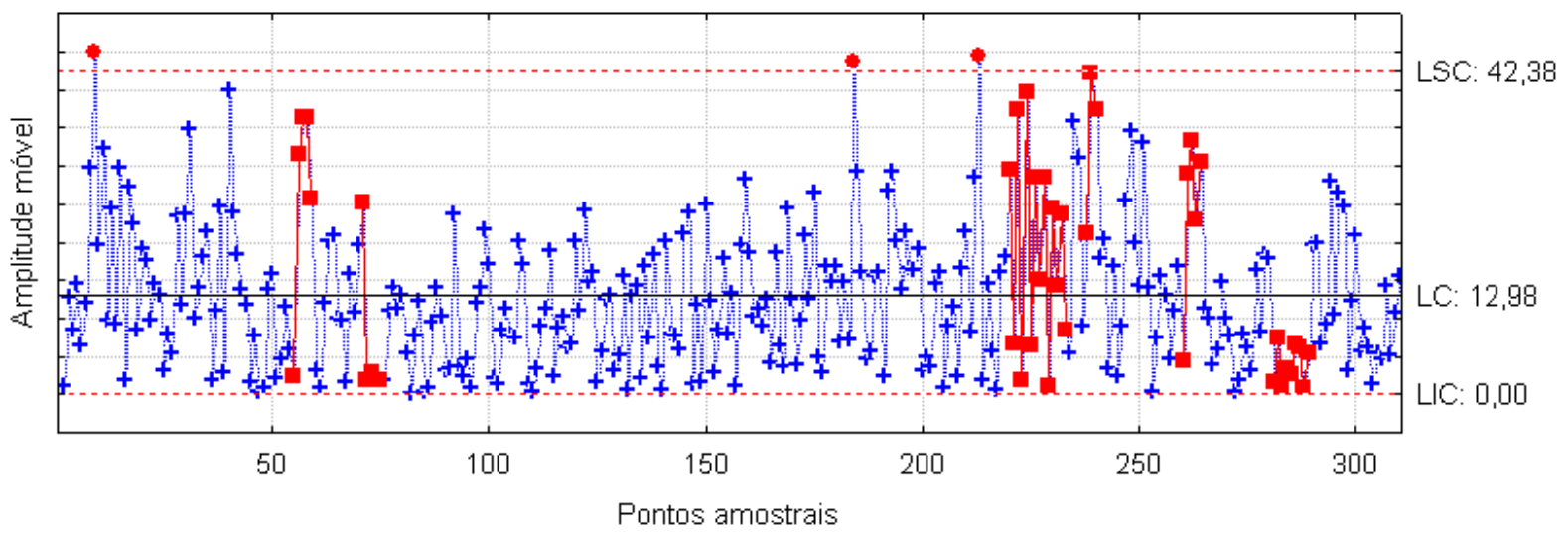


Figura 12 - Gráfico $\bar{X}$ aplicado ao resíduo da RCV

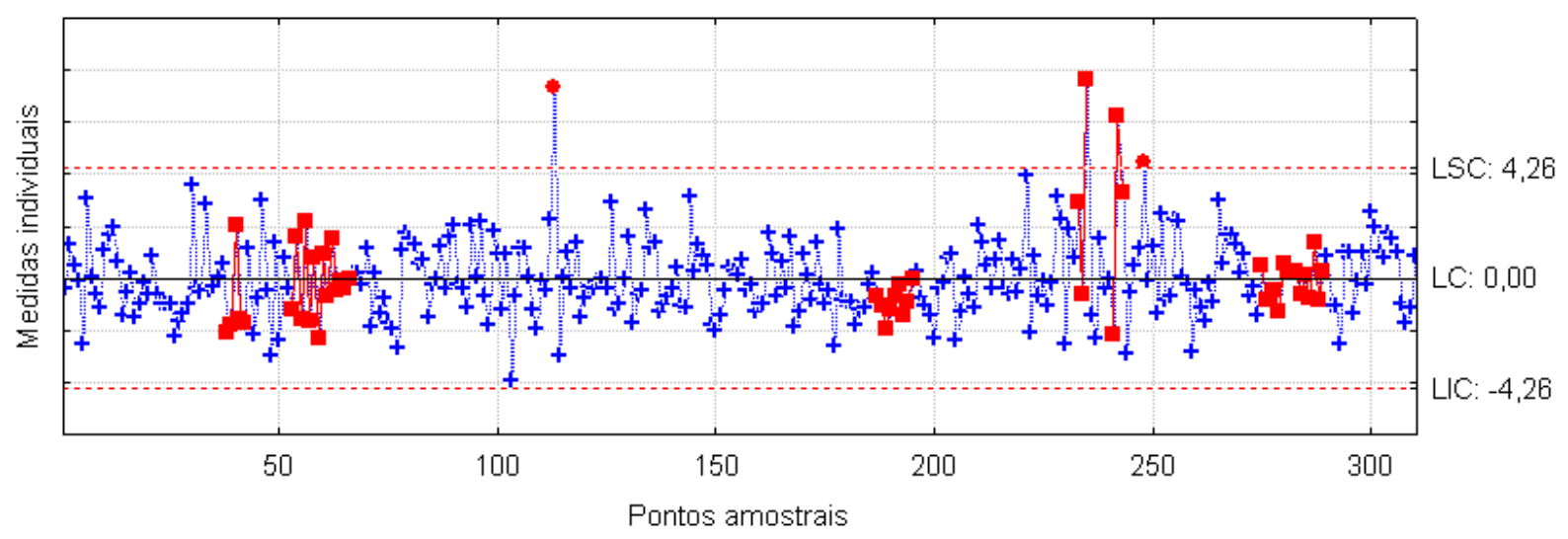

Figura 13 - Gráfico $\overline{M R}$ aplicado ao resíduo da RCV

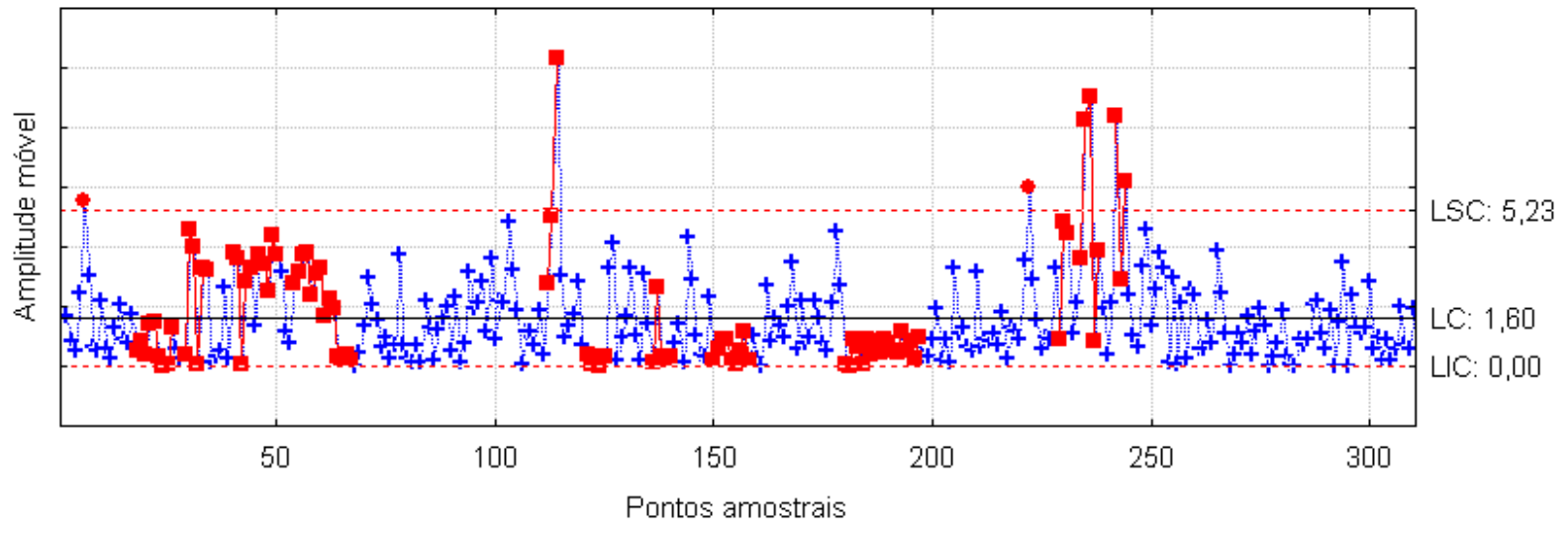

Analisando o gráfico $\bar{x}$ das Figuras 8, 10 e 12, constatou-se a existência de poucos pontos amostrais fora de controle se comparado ao gráfico $\bar{x}$ das séries originais apresentado nas Figuras 2, 4 e 6, respectivamente. Isso indica que o modelo de regressão dinâmica multivariada foi capaz de captar as variações que havia nas variáveis: teor de umidade, permeabilidade e RCV, e também foi eficiente em modelar o processo da Empresa A, em que o efeito da autocorrelação serial foi tratado. 


\subsection{Comentários gerais da análise}

Mediante a metodologia empregada, foi possível monitorar o comportamento do processo de fundição de moldagem em areia verde, utilizando o modelo de regressão dinâmica multivariada, que possibilita uma estimação conjunta das variáveis que compõem uma das etapas críticas de tal processo.

A modelagem realizada não teve apenas o objetivo de apresentar o valor futuro para cada uma das variáveis analisadas, a qual será útil para a empresa, mas também propiciou a detecção de pontos amostrais fora dos limites de controle, definindo se esses pontos são alarmes falsos ou não.

Com relação aos resultados obtidos após a aplicação dos gráficos de controle $\bar{X}$, apresentam-se as seguintes considerações para cada uma das variáveis:

Nos dados originais da variável teor de umidade haviam vinte e seis pontos amostrais fora dos limites de controle; após o ajuste do modelo de regressão dinâmica multivariada, constataram-se apenas dois pontos amostrais fora dos limites de controle.

Nos dados originais da variável permeabilidade haviam catorze pontos amostrais fora dos limites de controle; após o ajuste do modelo de regressão dinâmica multivariada, constatou-se a existência de dois pontos amostrais fora dos limites de controle.

Nos dados originais da variável RCV haviam vinte pontos amostrais fora dos limites de controle; após o ajuste do modelo de regressão dinâmica multivariada, constatou-se a existência de quatro pontos amostrais fora dos limites de controle.

Investigações conduzidas no processo produtivo revelam que, após o ajuste dos dados por meio do modelo de regressão dinâmica multivariada, o efeito da autocorrelação foi tratado e que os pontos amostrais que existiam nos gráficos de controle referentes aos dados originais realmente servem como um alerta pela característica do processo. Desse modo, o modelo de regressão dinâmica multivariada evidenciou uma melhora significativa na detecção de pontos amostrais fora de controle, tendo em vista que, o comportamento das variáveis pode estar influenciando umas às outras, minimizando os alarmes falsos no processo. 
Com os resultados obtidos, pode-se afirmar que a variável teor de umidade é a mais instável em relação às outras variáveis estudadas, pois apresentou mais pontos amostrais fora dos limites de controle. Assim, pretende-se alertar a equipe de produção a fazer os ajustes necessários para obter um processo o mais próximo possível da especificação previamente estabelecida.

\section{CONSIDERAÇÕES FINAIS}

A presente pesquisa possui caráter relevante no setor industrial por apresentar uma metodologia alternativa às técnicas tradicionais de controle estatístico de qualidade.

Após a execução das etapas metodológicas conclui-se que, o procedimento proposto, de primeiro retirar a autocorrelação serial dos dados para depois ajustálos, pois todos os processos produtivos avaliados apresentavam-se autocorrelacionados, mostrou-se adequado estatisticamente.

Os resultados apontam que o objetivo geral proposto de modelar processos produtivos industriais autocorrelacionados com o fim de analisar a estabilidade desses processos foi alcançado, sendo de fundamental importância para as pessoas envolvidas diretamente no processo como forma de fornecer informações gerenciais quanto à ação de medidas corretivas, possibilitando alcançar a melhoria contínua.

No caso específico desta pesquisa, optou-se por ajustar um modelo de regressão dinâmica por meio do estimador SUR para as variáveis referentes ao processo de fundição de moldagem em areia verde. Sob o qual foi possível detectar a presença de alarmes falsos nos dados originais das variáveis estudadas.

Buscou-se, através do estudo proposto, evidenciar possibilidades de redução de custos associados ao produto de má qualidade relacionado a falhas internas. Portanto, se as variáveis, em análise, estiverem fora de controle, ocasionarão um dispêndio ainda mais elevado de recursos financeiros, os quais deixarão de ser empregados em outros setores da empresa e em outras melhorias, seja de tecnologia ou de recursos humanos.

\section{AGRADECIMENTOS}


Os autores agradecem aos revisores anônimos pelas contribuições pertinentes a pesquisa e a empresa que gentilmente cedeu os dados.

\section{REFERÊNCIAS}

ALWAN, L. C.; ROBERTS, H. V. Times series modeling for statistical process control. Journal of Business \& Economics Statistics, v. 6, n. 1, p. 87-95, 1988.

BAYER, F. M.; VICINI, L.; SOUZA, A. M. Modelo de vetores autoregressivos no monitoramento do preço do boi gordo: uma ferramenta auxiliar na tomada de decisão. In: XXVII Encontro Nacional de Engenharia de Produção, 17, Foz do Iguaçu, 2007. Anais ... Foz do Iguaçu: Associação Brasileira de Engenharia de Produção, 2007, p. 1-9.

BAYER, F. M.; SOUZA, A. M. Modelagem e previsão da taxa de ocupação hospitalar por meio de equações simultâneas: uma ferramenta de gestão. GEPROS - Gestão da Produção, Operações e Sistemas, v. 3, p. 85-96, 2007.

BRONS, M.; NIJKAMP, P.; PELS, E.; RIETVELD, P. A meta-analysis of the price elasticity of gasoline demand: a SUR approach. Energy Economics, v. 30, n. 5, p. 2105-2122, sep., 2008.

CHAREMZA, W. W.; DEADMAN, D. New directions in econometric practice general to specific modelling, cointegration and vector autoregression. 2.ed, Cheltenham: Edward Elgar Publishier Limited, 1997.

CLARO, F. A. E.; COSTA, A. F. B.; MACHADO, M. A. G. Gráficos de controle de EWMA e de X-barra para monitoramento de processos autocorrelacionados.

Revista Produção, São Paulo, v. 17, n. 3, p. 536-546, 2007.

CREEL, M; FARELL, M. SUR estimation of multiple time-series models with heteroscedasticity and serial correlation of unknown form. Economics Letters, v. 53, n. 3, p. 239-245, dec., 1996.

GATU, C; KONTOGHIORGHES, E. J. Estimating all possible SUR models with permuted exogenous data matrices derived from a VAR process. Journal of Economic Dynamics and Control, v. 30, n. 5, p. 721-739, may., 2006.

GUJARATI, D. N. Econometria básica. São Paulo: Makron Books, 2000.

HAMILTON, J. Time series analysis. New Jersey: Princeton University Press, 1994.

HARRIS, T. J.; ROSS, W. H. Statistical process control procedures for correlated observations. The Canadian Journal of Chemical Engineering, v. 69, p. 48-57, 1991.

HILL, C.; GRIFFITHS, W.; JUDGE, G. Econometria. São Paulo: Saraiva, 1999. 
LÜTKEPOHL, H. Introduction to multiple time series analysis. 2.ed, Berlin: Springer-Verlag, 1991.

MADDALA, G. S. Introduction to econometrics. 2.ed, New Jersey: Prentice Hall, 1992.

MINGOTI, S. A.; YASSUKAWA, F. R. S. Uma comparação de gráficos de controle para a média de processos autocorrelacionados. Revista Eletrônica Sistemas \& Gestão, v. 3, n. 1, p. 55-73, 2008.

MONTGOMERY, D.C. Introduction to statistical quality control. 3.ed. New York: John Wiley \& Sons, Inc., 1997.

MONTGOMERY, D. C. Introdução ao controle estatístico da qualidade. 4.ed, Rio de Janeiro: LTC, 2004.

MOREIRA JUNIOR, F. J.; TEN CATEN, C. S. Estudo sobre o efeito da autocorrelação de modelos AR(1) no controle estatístico de processo. In: XXIV ENCONTRO NACIONAL DE ENGENHARIA DE PRODUÇÃO, 14, Florianópolis, 2004. Anais ... Florianópolis: Associação Brasileira de Engenharia de Produção, 2004, p. 1705-1712.

MUROLO, A. C.; SILVA, E. M. da; SILVA, E. M. da.; GONÇALVES, V. Estatística: para os cursos de Economia, Administração e Ciências Contábeis. São Paulo: Atlas, 1999.

RAMOS, A. W.; HO, L. L. Procedimentos inferenciais em índices de capacidade para dados autocorrelacionados via bootstrap. Revista Produção, São Paulo, v. 13, n. 3, p. 50-62, 2003.

REINSEL, G. C. Elements of multivariate time series analysis. New York: Springer-Verlag, 1993.

RUSSO, S. L.; CAMARGO, M. E.; SAMOHYL, R. W. Gráficos de controle baseado nos resíduos do modelo de regressão Poisson. Revista Produção Online, v. 8, n. 4, dez., 2008.

SALLES, A. A. Análise multivariada do risco sistemático dos principais mercados de ações da América Latina: um enfoque bayesiano. In: XXVI ENCONTRO NACIONAL DE ENGENHARIA DE PRODUÇÃO, 16, Fortaleza, 2006. Anais ... Fortaleza: Associação Brasileira de Engenharia de Produção, 2006, p. 1-9.

SILVA, W. V.; FONTANINI, C. A. C.; DEL CORSO, J. M. Garantia da qualidade do café solúvel com o uso do gráfico de controle de somas acumuladas. Revista Produção Online, v. 7, n. 2, p. 43-63, ago., 2007.

SILVEIRA, E. F.; WERNER, L. Proposta de método de priorização de processos a serem monitorados no controle estatístico de processo: uma aplicação em trocador de calor. Revista Produção Online, v. 11, n. 1, p. 116-135, mar., 2011. 
SOUZA, A. M.; SAMOHYL, R. W.; MALAVÉ, C. O. Multivariate feedback control: an application in a productive process. Computers \& Industrial Engineering, v. 46, p. 837-850, 2004a.

. Aplicação de um modelo paramétrico multivariado para o controle da temperatura de fornos de túnel. Revista Produção, São Paulo, v. 14, n. 2, p. 82-94, 2004b.

SOUZA, A. M.; SOUZA, F. M.; MENEZES, R. Procedure to evaluate multivariate statistical process control using ARIMA-ARCH Models. Journal of Japan Industrial Management Association, v. 63, n.2E, p.112-123, 2012.

TSAI, T.; CHIANG, Y.; WU, S. Conditional maximum likelihood estimation for control charts in the presence of correlation. Brazilian Journal of Probability and Statistics, v. 18, n. 2, p. 151-162, dec., 2004.

ZELLNER, A.; THEIL, H. Three-stage least squares: simultaneous estimation of simultaneous equations. Econometrica, v. 30, n. 1,p. 54-78, jan., 1962.

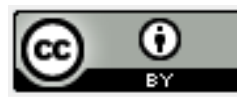

Artigo recebido em 30/01/2012 e aceito para publicação em 07/11/2013. 